\title{
QUADERNI Quaderni
}

Communication, technologies, pouvoir

99-100 | Hiver 2019-2020

Technologies et jeux de pouvoir

\section{Yves-Marie Bercé, Esprits et Démons. Histoire des phénomènes d'hystérie collective}

\section{Véronique Campion-Vincent}

\section{(2) OpenEdition}

1 Journals

Édition électronique

URL : https://journals.openedition.org/quaderni/1592

DOI : 10.4000/quaderni.1592

ISSN : 2105-2956

Éditeur

Les éditions de la Maison des sciences de l'Homme

Édition imprimée

Date de publication : 5 janvier 2020

Pagination : 169-172

\section{Référence électronique}

Véronique Campion-Vincent, "Yves-Marie Bercé, Esprits et Démons. Histoire des phénomènes d'hystérie collective », Quaderni [En ligne], 99-100 | Hiver 2019-2020, mis en ligne le 05 janvier 2020, consulté le 04 janvier 2023. URL : http://journals.openedition.org/quaderni/1592 ; DOI : https://doi.org/10.4000/ quaderni.1592 


\section{Compte rendu}

\section{Esprits et Démons. Histoire des phénomènes d'hystérie collective Yves-Marie Bercé}

La librairie Vuibert, Paris, 2018

par Véronique Campion-Vincent

FMSH - Paris

1. Le Roi caché. Sauveurs et imposteurs. Mythes politiques populaires dans l'Europe moderne, Paris : Fayard, 1990.

2. «Les semeurs de peste » pp. 85-94 in La vie, la mort, la foi, le temps. Mélanges offerts à Pierre Chaunu, Paris: Presses Universitaires de France, 1993.

3. Le chaudron et la lancette : croyances populaires et médecine préventive, 1798-1830, Paris : Presses de la Renaissance, 1998.

4. À la découverte des trésors cachés : du $\mathrm{XVI}$ siècle à nos jour, Paris : Perrin, 2004.
Voici un livre à la fois ambitieux et modeste, écrit par un historien de la période moderne (XVI ${ }^{\mathrm{e}} \mathrm{XVIII}{ }^{\mathrm{e}}$ siècles). Yves-Marie Bercé (Membre de l'Institut, Académie des Inscriptions et Belles Lettres, 2007) a beaucoup étudié les révoltes liées à la mise en place de l'absolutisme en France. Il a également fait preuve d'une grande curiosité pour les sujets « hors-piste», étudiant par exemple la figure légendaire et mythique du Roi caché ${ }^{1}$, les accusations visant les semeurs de peste ${ }^{2}$, les réactions savantes et populaires à l'introduction de la vaccination ${ }^{3}$ ou la quête des trésors cachés ${ }^{4}$ en tenant toujours à distance les assertions péremptoires de « rationalisation » qui abondent dans les études sur ces champs marginaux.

Ici Yves-Marie Bercé reprend les récits et présente ses analyses de multiples cas d' « hystéries collectives », c'est-à-dire des explosions de comportements anormaux et spectaculaires (convulsions incontrôlables ou vomissements incoercibles par exemple) très contagieux, concernant majoritairement des femmes et souvent expliqués par l'action d'esprits malfaisants. Les possessions diaboliques, avec l'aide d'affidés des démons, sont fréquemment affirmées. Si au XXI ${ }^{\mathrm{e}}$ siècle ces explosions sont plutôt désignées comme des « psychoses collectives », l'auteur a cependant voulu conserver au travers de l'expression « hystéries collectives » le vocabulaire et les débats d'idées ayant eu cours au moment des faits étudiés.

La majeure partie de l'ouvrage est consacrée à l'Europe du $\mathrm{XVI}^{\mathrm{e}}$ au $\mathrm{XIX}^{\mathrm{e}}$ siècle (pages 21-206). Les quatre premiers chapitres dressent un état des lieux factuel : Possessions célèbres des couvents français du XVII ${ }^{\mathrm{e}}$ siècle, Aixen-Provence (1611) puis Loudun (1632-1637) où à la suite d'une religieuse 
accusatrice s'affirmant possédée tout un couvent s'enflamme et les ecclésiastiques désignés comme sorciers (Gaufridy à Aix-en-Provence et Grandier à Loudun) montent sur le bûcher. Explosions collectives comme expressions des dissidences religieuses des petits prophètes protestants des Cévennes en 1680-1704, puis dans les années 1730 des jansénistes avec le cimetière SaintMédard envahi de " convulsionnaires » venus vénérer la tombe du diacre Pâris où se produisaient de multiples guérisons miraculeuses. Zoom sur les permanences des crises de possession dans les pays alpins, depuis Charles Borromée (archevêque de Milan au XVI ${ }^{\mathrm{e}}$ siècle) face aux « inspirités » autour du lac de Côme en 1582, jusqu'aux épisodes collectifs de Morzine où des petites-filles et jeunes femmes se disent possédées (1857-1865) et ceux du Frioul (1878). Enfin, le cas du complexe du tarentisme sicilien et des thérapies par la musique et la danse qui le caractérisent ${ }^{5}$.

Trois chapitres plus analytiques viennent ensuite. Tout d'abord, l'auteur analyse les interactions entre possession et exorcisme. Yves-Marie Bercé note que les consignes des autorités ecclésiastiques d'un exorcisme discret n'étaient pratiquement jamais observées et les exorcismes publics se transformaient en théâtre du sensationnel suivi avec gourmandise par le public qui renforçait les possédées dans leurs ébats spectaculaires, dramatiques mais également souvent comiques. Ensuite, il présente les cas liés aux asiles d'orphelins ou de pauvres et aux institutions scolaires où éclatent des épidémies de possession. Aux Pays-Bas, trois cas, aux $\mathrm{XVI}^{\mathrm{e}}, \mathrm{XVII}^{\mathrm{e}}$ et XVIII ${ }^{\mathrm{e}}$ siècles, ont été l'objet d'investigations savantes qui se sont conclues par la dispersion des enfants. Des faits voisins se retrouvent dans les dernières décennies du XX $\mathrm{XX}^{\mathrm{e}}$ siècle. En 1983, toute une école de jeunes filles (727) située dans une ville de la province d'Hébron en Palestine ressent des malaises violents que l'opinion publique explique par des gaz toxiques qui auraient été répandus par l'armée israélienne occupante. Une autre explosion épidémique surgira en 1993 en Égypte et atteindra le Kosovo où les enfants albanophones accusent l'armée serbe d'empoisonnement. Enfin, la condition féminine de cette époque est évoquée à travers les débats autour de l'hystérie dont la causalité psychogénique (et non pas liée à une sexualité réprimée) est affirmée dès la fin du XVII ${ }^{\mathrm{e}}$ siècle par certains médecins, la condition nerveuse des « vapeurs » soignées par des sels provoquant des éternuements qui expulsent les maux et le rôle des isolats féminins - ceux des couvents mais aussi ceux des communautés paysannes montagnardes où les hommes partent chercher du travail en plaine l'été ou l'hiver. 
Les aires non-européennes sont à leur tour abordées dans les deux derniers chapitres de l'ouvrage. Les conceptions spirites des populations d'Afrique ont amené à la création des nouvelles religions des Africains transplantés par l'esclavage en Haïti (vaudou), à Cuba (santeria) et au Brésil (candomblé). Yves-Marie Bercé traite également de l'influence de ces conceptions spirites en Tunisie comme au Maroc où l'on retrouve la thérapie musicale avec la musique des Gnaouas invoquée par ceux et celles qui se pensent victimes de maléfices. Enfin le dernier chapitre décrit l'évolution des transes dans les populations de l'extrême nord scandinave, désignées comme Lapons puis comme Sames. Avec les conversions au christianisme, les chamans païens traditionnels sont désignés comme des sorciers ayant pactisé avec le diable. Puis, au XIX $\mathrm{X}^{\mathrm{e}}$ siècle, des épisodes de rigorisme piétiste conduisent une quête passionnée du mal qui mène à des crises collectives avec « parler en langues » et convulsions. En Norvège, un dernier épisode meurtrier, influencé par un pasteur passionné, sera puni par la justice avec deux exécutions. Une concise conclusion (pages 241-249) clôt enfin l'ouvrage.

Dans le cadre de ce compte rendu, on se contentera de quelques remarques sans prétendre en épuiser les pistes stimulantes.

Au-delà des diversités temporelles, spatiales et culturelles, Yves-Marie Bercé détecte « l'unité des espérances humaines et de la dynamique d'imitation » (p. 9). Espérances déçues, désirs inassouvis, persécutions, isolement, etc. présentent une collection d' « impasses psychiques» dont les victimes s'évadaient par certains jeux de rôle proposés par les rites collectifs régnants, jeux de conjuration de l'angoisse dont les « modèles de fuite rituelle semblent disponibles comme dans un répertoire dramatique » (pages 248, 249).

Si l'Europe des Temps Modernes « a été traversée par une vague d'obsession diabolique » (p. 21), les épisodes de psychoses collectives entraînaient des débats entre les élites opposant la croyance aux méfaits du Malin (chez les exorcistes en particulier mais surtout dans «l'opinion publique » souvent à l'origine de la judiciarisation des affaires) aux attitudes sceptiques de plus en plus répandues chez les hauts magistrats. Subi comme d'origine diabolique, l'accès à la transe peut aussi être recherché comme signe d'élection divine. C'est le cas pour les dissidences religieuses au XVIII ${ }^{\mathrm{e}}$ siècle (petits prophètes cévenols, convulsionnaires jansénistes de Saint-Médard) ou pour les cultes d'origine africaine. 
Cependant, ces phénomènes qui a priori nous semblent aujourd'hui archaïques, le sont-ils véritablement dans la France du XXI ${ }^{\mathrm{e}}$ siècle ? Le commerce avec les esprits, de la Nature ou des Anciens demeure dans certains cercles d'inspiration ésotériste un idéal recherché et, dans ces milieux en quête spirituelle, on s'efforce à « devenir chaman » par la méditation collective, mais surtout par l'ingestion de drogues utilisées dans les cultures traditionnelles faisant une place au chamanisme telles l'iboga (Gabon) ou l'ayahuasca (Pérou $)^{6}$. Certes marginales, ces quêtes ont toutefois donné lieu à une véritable industrie « touristique » de l'Europe vers ces pays. Le phénomène n'est d'ailleurs nullement limité à la France et par exemple la place du chamanisme est fort importante en Russie 7 . Cependant, dans d'autres couches des populations vivant en France, l'explication de désordres par l'action d'esprits et la causalité diabolique, certes moins prégnante, n'a pas disparu, ainsi dans les cultes évangéliques en plein essor qui prétendent parfois guérir des possessions diaboliques.

Un mot sur la présentation du livre : sa lecture est rendue aisée par l'absence de ces références aux auteurs cités qui alourdissent tant d'ouvrages. Celles-ci sont bien citées, mais dans une section « Références » (pages 251-266) qui précède une copieuse « Bibliographie » (pages 267-282). Un lecteur très attentif relèvera certes dans la section « Références » des auteurs non repris dans la « Bibliographie » mais on félicitera l'auteur d'avoir adopté ce système qui permet une vraie concentration du corps de l'ouvrage sans renoncer à la précision des références étudiées.

\footnotetext{
6. Jan Kounen,

Vincent Ravalec et Jeremy Nardy. Plantes $\&$ chamanisme Conversations autour de l'ayahuasca \& de l'iboga Paris: Mama Éditions [Chamanismes], 2012.

7. Valentina Kharitonova "Revived Shamanism in the Social Life of Russia" Folklore.ee, vol. 62, 27-54.

Le tourisme vers l'ayahuasca est évoqué p. 48 : www.folklore.ee/folklore/ vol62/kharitonova.pdf
} 